\title{
On the Langberg-Médard Multiple Unicast Conjecture
}

\author{
Kai Cai • Guangyue Han
}

Received: date / Accepted: date

\begin{abstract}
A version of the multiple unicast conjecture, proposed by M. Langberg and M. Médard [4], says that, there exists an undirected fractional multicommodity flow, or simply, multi-flow, with rate $(1,1, \cdots, 1)$ for strongly reachable networks. In this paper, we propose a new type of matrix optimization problem to attack this conjecture: By giving upper and lower bounds on its solution, we prove that there exists a multi-flow with rate $\left(\frac{8}{9}, \frac{8}{9}, \cdots, \frac{8}{9}\right)$ for such networks; on the other hand though, we show that the rate of any multi-flow constructed using this framework cannot exceed $(1,1, \cdots, 1)$.
\end{abstract}

\section{Introduction}

Let $\mathcal{N}=(V, A, S, R)$ be a directed network with underlying digraph $D=$ $(V, A)$, senders $S=\left\{s_{1}, s_{2}, \ldots, s_{k}\right\} \subseteq V$ and receivers $R=\left\{r_{1}, r_{2}, \ldots, r_{k}\right\} \subseteq$ $V . \mathcal{N}$ is said to be fully reachable if there exists an $s_{i}-r_{j}$ directed path $P_{s_{i}, r_{j}}$ for each $i, j=1,2, \cdots, k$, and strongly reachable if, further, for each $j=$ $1,2, \cdots, k$, the paths $P_{s_{1}, r_{j}}, P_{s_{2}, r_{j}}, \cdots, P_{s_{k}, r_{j}}$ are edge-disjoint. Let $\overline{\mathcal{N}}$ denote the underlying undirected network of $\mathcal{N}$, where the orientation in $\mathcal{N}$ is ignored. Throughout this paper, we assume that each edge in $\overline{\mathcal{N}}$ is of unit capacity.

The multiple unicast conjecture [1] [2], which turned out to be one of the hardest problems in the theory of network coding [3], has been open for more than one decade. A weaker version of this conjecture, proposed by M. Langberg and M. Médard [4], can be stated in the graph-theoretic language as follows.

This research is partly supported by a grant from the University Grants Committee of the Hong Kong Special Administrative Region, China (Project No. AoE/E-02/08)

Kai Cai

Department of Mathematics, The University of Hong Kong, Pokfulam Road, Hong Kong. E-mail: kcai@hku.hk

Guangyue Han

Department of Mathematics, The University of Hong Kong, Pokfulam Road, Hong Kong. E-mail: ghan@hku.hk 
Conjecture 1 If $\mathcal{N}$ is strongly reachable, then there exists a feasible $\left(s_{1}, s_{2}, \ldots, s_{k}\right)$ $\left(r_{1}, r_{2}, \ldots, r_{k}\right)$ multi-flow with rate $(1,1, \cdots, 1)$ in $\overline{\mathcal{N}}$.

If this conjecture is true, then it gives a new type of sufficient conditions on the existence of multi-flows, which is almost invariably characterized by the cut condition in the existing literature [5]. By using a flow construction method, the authors of [4] obtained a feasible multi-flow with rate $\left(\frac{1}{3}, \frac{1}{3}, \cdots, \frac{1}{3}\right)$. In this paper, we extend their method to construct a feasible multi-flow with rate $\left(\frac{8}{9}, \frac{8}{9}, \cdots, \frac{8}{9}\right)$; furthermore, we prove that the rate of any feasible multi-flow constructed using our framework cannot exceed $(1,1, \cdots, 1)$. Our results is obtained by analysing the upper and lower bounds of a matrix optimization problem, which may be of independent interest in its own right.

The rest of the paper is organized as follows. In Section II, we introduce the flow construction method. A matrix optimization problem is proposed in section III. Finally, the lower and upper bounds on the proposed problem are investigated in Sections IV and V, respectively.

\section{The Flow Construction Method}

In this section, we first introduce some terminologies and results in the theory of multi-flows. Then, in the second subsection, we introduce the flow construction method. In the third subsection, we discuss the feasibility for the constructed multi-flows.

\subsection{Multi-Flow Basics}

Consider a directed network $\mathcal{N}=(V, A, S, R)$ and its undirected version $\overline{\mathcal{N}}$. For an $\operatorname{arc} a=(u, v) \in A$, we call $u$ the tail of $a$, denoted by tail $(a)$, and $v$ the head of $a$, denoted by head $(a)$. For any $v_{0}, v_{n} \in V$, a $v_{0}-v_{n}$ directed path in $\mathcal{N}$ is a sequence $\left(v_{0}, a_{1}, v_{1}, a_{2}, \ldots, a_{n}, v_{n}\right)$, such that all $v_{i}$ are distinct, and $\operatorname{tail}\left(a_{i}\right)=v_{i-1}$ and $h e a d\left(a_{i}\right)=v_{i}$ for all $i$. Slightly abusing the notation, for an arc $a$ and a directed path $P$ in $\mathcal{N}$, we use $a \in P$ to mean that $a$ occurs on $P$.

For any $s, r \in V$, an $s-r$ flow is a function $f: A \rightarrow \mathbb{R}$ satisfying the following flow conservation law:

$$
\operatorname{excess}_{f}(v)=0, \quad \forall v \notin\{s, r\}
$$

where

$$
\operatorname{excess}_{f}(v):=\sum_{a \in A: \operatorname{head}(a)=v} f(a)-\sum_{a \in A: \operatorname{tail}(a)=v} f(a) .
$$

It is easy to see that $\left|\operatorname{excess}_{f}(s)\right|=\left|\operatorname{excess}_{f}(r)\right|$, which is called the value (or rate) of $f$.

Note that the above definitions naturally give rise to a undirected flow, i.e., a fractional flow of $\overline{\mathcal{N}}$, and it is not needed to differentiate an $s$-r flow 
from an $r$-s flow. This is different from Schrijver [5], where a flow must be a non-negative function.

There are two kinds of operations on the flows defined as above. Firstly, the set of all $s-r$ flows naturally forms a linear space over $\mathbb{R}$; particularly, for any two $s$ - $r$ flows $f_{1}, f_{2}$ and scalars $k_{1}, k_{2} \in \mathbb{R}$, and the function $f=k_{1} f_{1}+k_{2} f_{2}$ is again an $s-r$ flow. Secondly, let $f$ be an $s$ - $t$ flow and $g$ be a $t-r$ flow such that

$$
\operatorname{excess}_{f}(t)=-\operatorname{excess}_{g}(t) \text {. }
$$

Then by definition, $f+g$ is an $s-r$ flow, which is called the concatenation of $f$ and $g$. Adopting the notational convention in defining the concatenation of paths in [5], the concatenation of $f$ and $g$ will be denoted by $f g$.

An $\left(s_{1}, s_{2}, \ldots, s_{k}\right)-\left(r_{1}, r_{2}, \ldots, r_{k}\right)$ multi-flow refers to a set of $k$ flows $\mathcal{F}=$ $\left\{f_{i}: i=1,2, \ldots, k\right\}$, where each $f_{i}$ is an $s_{i}-r_{i}$ flow. We say $\mathcal{F}$ has rate $\left(d_{1}, d_{2}, \ldots, d_{k}\right)$, where $d_{i}:=\mid$ excess $_{f_{i}}\left(s_{i}\right) \mid ;$ and, for any given $a \in A$, we define $|\mathcal{F}|(a)$ as

$$
|\mathcal{F}|(a):=\sum_{1 \leq i \leq k}\left|f_{i}(a)\right|
$$

The multi-flow $\mathcal{F}=\left\{f_{i}: i=1,2, \ldots, k\right\}$ is said to be feasible with respect to capacity function $c$ if $|\mathcal{F}|(a) \leq c(a)$ for all $a \in A$. Note that when $k=1$, the multi-commodity flow is a flow $f$, and we call $f$ feasible if $|f(a)| \leq c(a)$ for all $a \in A$. Recall that $c(a) \equiv 1$ in this paper.

\subsection{Flow Constructed from the Elementary Flows}

Let $\mathcal{N}$ be a fully reachable network and $\mathbf{P}=\left\{P_{s_{i}, r_{j}}\right\}_{i, j=1}^{k}$ be a set of $s_{i}-r_{j}$ directed paths of $\mathcal{N}$. For each $P_{s_{i}, r_{j}} \in \mathbf{P}$, define an $s_{i}-r_{j}$ flow as follows:

$$
f_{i, j}(a)=\left\{\begin{array}{l}
1, a \in P_{s_{i}, r_{j}}, \\
0, \text { otherwise. }
\end{array}\right.
$$

Let

$$
\mathbf{F}=\left\{f_{i, j} \mid 1 \leq i, j \leq k\right\} .
$$

In this paper, $\mathbf{P}$ and $\mathbf{F}$ will be referred to as a set of elementary paths and elementary flows of $\mathcal{N}$, respectively.

Before proceeding further, we clarify that by "the flow constructed from the elementary flows F", we mean the flow is obtained by a sequence of "operations," i.e., linear combinations and concatenations on the flows within $\mathbf{F}$. For example, $\frac{1}{3}\left[\left(f_{1,2}-f_{3,2}\right) f_{3,1}\right]$ is an $s_{1}-r_{1}$ flow constructed from $\mathbf{F}$ with value $\frac{1}{3}$. Note that to construct a new $s_{i}-r_{j}$ flow from $\mathbf{F}$, we only need to ensure that the flow conservation law is satisfied by all the senders except $s_{i}$ and all the receivers except $r_{j}$.

Now, we arrange the elementary flows into a matrix $F$, say,

$$
F=\left(\begin{array}{cccc}
f_{1,1} & f_{1,2} & \cdots & f_{1, k} \\
f_{2,1} & f_{2,2} & \cdots & f_{1, k} \\
\vdots & \vdots & \vdots & \vdots \\
f_{k, 1} & f_{k, 2} & \cdots & f_{k, k}
\end{array}\right) .
$$


Let

$$
C=\left(\begin{array}{cccc}
c_{1,1} & c_{1,2} & \cdots & c_{1, k} \\
c_{2,1} & c_{2,2} & \cdots & c_{1, k} \\
\vdots & \vdots & \vdots & \vdots \\
c_{k, 1} & c_{k, 2} & \cdots & c_{k, k}
\end{array}\right)
$$

be an arbitrary $k \times k$ matrix with $c_{i, j} \in \mathbb{R}$ and let

$$
f=C \circ F=\sum_{i, j=1}^{k} c_{i, j} f_{i, j},
$$

where $C \circ F$ denotes the formal Hadmard product of $C$ and $F$. Then, we have:

Theorem $1 f$ is an $s_{i_{0}}-r_{j_{0}}$ flow constructed from $\mathbf{F}$ with rate $d$ if and only if the following conditions are satisfied:

1. $\sum_{\ell=1}^{k} c_{i, \ell}=0$, for all $1 \leq i \leq k, i \neq i_{0}$;

2. $\sum_{\ell=1}^{k} c_{\ell, j}=0$, for all $1 \leq j \leq k, j \neq j_{0}$;

3. $\sum_{i=1}^{k} \sum_{j=1}^{k} c_{i, j}=d$.

Proof Note that excess $f\left(s_{i}\right)=\sum_{\ell=1}^{k} c_{i, \ell}$ and excess $_{f}\left(r_{j}\right)=\sum_{\ell=1}^{k} c_{\ell, j}$. Condition 1) implies that the conservation law is satisfied by all the senders except $s_{i_{0}}$; Condition 2) implies that it is satisfied by all the receivers except $r_{j_{0}}$; Condition 3) implies that the value of $f$ is $d$.

Note that under Conditions 1) and 2), Condition 3) of Theorem 1 is equivalent to

$\left.3^{\prime}\right) \sum_{\ell=1}^{k} c_{i_{0}, \ell}=d$, or

$\left.3^{\prime \prime}\right) \sum_{\ell=1}^{k} c_{\ell, j_{0}}=d$, or

$\left.3^{\prime \prime \prime}\right) \sum_{\ell=1}^{k} c_{i_{0}, \ell}=\sum_{\ell=1}^{k} c_{\ell, j_{0}}=d$.

Let $f=C \circ F$ be a flow constructed from $\mathbf{F}$, we call $C$ the coefficient matrix of $f$. By Theorem 1, there is a one-to-one correspondence from the flows constructed from $\mathbf{F}$ with rate $d$ to the matrices satisfying Conditions 1),2) and 3). This correspondence can be directly applied to the multi-flow: Let $\mathcal{F}=\left\{f_{1}, f_{2}, \cdots, f_{k}\right\}$ be an $\left(s_{1}, s_{2}, \cdots, s_{k}\right)-\left(r_{1}, r_{2}, \cdots, r_{k}\right)$ multi-flow constructed from $\mathbf{F}$ with rates $(d, d, \cdots, d)$, then there exist a tuple of matrices $\left(C_{1}, C_{2}, \cdots, C_{k}\right)$ such that 1$)$ the sum of elements in $j$-th column and the sum of elements in $j$-th column of $C_{i}$ are all equal 0 when $j \neq i$;2) the sum of all elements in $C_{i}$ are all equal $d$, for $i=1,2, \cdots, k$. Note that we sometimes identify $\left(C_{1}, C_{2}, \cdots, C_{k}\right)$ with the corresponding multi-flow.

From now on, we always suppose $\mathcal{F}=\left\{f_{1}, f_{2}, \cdots, f_{k}\right\}$ is a multi-flow constructed from the elementary flows $\mathbf{F}$ such that the coefficient matrix of $f_{\ell}$ is $\left(c_{i, j}^{(\ell)}\right) \in \mathbb{R}^{k \times k}$ for $\ell=1,2, \cdots, k$.

Definition 1 (Symmetric Multi-Flow) For each $\ell=1,2, \cdots, k$, if $\left(c_{i, j}^{(\ell)}\right)=$ $E_{1, \ell}\left(c_{i, j}^{(1)}\right) E_{1, \ell}$, then $\mathcal{F}$ is called a symmetric multi-flow, where $E_{i, j}$ is the elementary matrix formed by swapping the $i$-th row and $j$-th row of the $k \times k$ identity matrix. 
Definition 2 (Homogeneous Multi-Flow) Let $\mathcal{F}$ be a symmetric flow. If there exist $a, b \in \mathbb{R}$, such that

$$
c_{i, j}^{(1)}= \begin{cases}a, & i=j=1 \\ b, & i=1, j \neq 1 \text { or } j=1, i \neq 1 \\ -\frac{b}{k-1}, & \text { otherwise }\end{cases}
$$

Then, $\mathcal{F}$ is called a homogeneous multi-flow.

Obviously, a homogeneous multi-flow has rate $(d, d, \cdots, d)$ with $d=a+$ $(k-1) b$.

Example 1 ([4]) The mulit-flow $\mathcal{F}$ constructed in [4] is a homogeneous multiflow with

$$
a=\frac{2 k-3}{k(3 k-4)}, \quad b=\frac{k-1}{k(3 k-4)}
$$

and rate $(d, d, \cdots, d)$ with

$$
d=\frac{2 k-3}{k(3 k-4)}+\frac{(k-1)^{2}}{k(3 k-4)}=\frac{k^{2}-2}{3 k^{2}-4 k}>\frac{1}{3}
$$

\subsection{Feasibility of the Flow Constructed from $\mathbf{F}$}

In this section and thereinafter, we always assume that $\mathcal{N}=(V, A, S, R)$ is a strongly reachable network and $\mathbf{P}=\left\{P_{s_{i}, r_{j}}\right\}_{i, j=1}^{k}$ is a set of elementary paths such that $P_{s_{1}, r_{j}}, P_{s_{2}, r_{j}}, \cdots, P_{s_{k}, r_{j}}$ are edge-disjoint for each $j=1,2, \cdots, k$. For any $a \in A$, denote by $\mathcal{P}(a)$ the elementary paths passing through $a$. Suppose there are totally $\alpha(a)$ paths in $\mathcal{P}(a)$ and let

$$
\mathcal{P}(a):=\left\{P_{s_{i_{1}}, r_{j_{1}}}, P_{s_{i_{2}}, r_{j_{2}}}, \cdots, P_{s_{i_{\alpha(a)}}, r_{j_{\alpha(a)}}}\right\} .
$$

Lemma $1 j_{1}, j_{2}, \cdots, j_{\alpha(a)}$ are distinct.

Proof Note that, for a strongly reachable network, in the set $\mathbf{P}$ of elementary paths, the paths $P_{s_{1}, r_{j}}, P_{s_{2}, r_{j}}, \cdots, P_{s_{k}, r_{j}}$ are edge-disjoint for any $1 \leq j \leq k$, which immediately implies the lemma.

We shall call the set of integers $\left\{j_{1}, j_{2}, \cdots, j_{\alpha(a)}\right\}$ the support of a, denoted by Support(a). Now, we define the coordinate of an arc $a$ as follows.

Definition 3 (Coordinate) Suppose $\mathcal{P}(a):=\left\{P_{s_{i_{1}}, r_{j_{1}}}, P_{s_{i_{2}}, r_{j_{2}}}, \cdots, P_{s_{i_{\alpha(a)}}, r_{j_{\alpha(a)}}}\right\}$. Then, the coordinate of $a$ is a vector $x=\left(x_{1}, x_{2}, \cdots, x_{k}\right)$ such that for $\ell=1,2, \cdots, k$,

$$
x_{\ell}=\left\{\begin{array}{l}
i_{m}, \text { if } \ell=j_{m} \in \operatorname{Support}(a), \\
0, \quad \ell \notin \operatorname{Support}(a) .
\end{array}\right.
$$

We also use Support( $x)$ or Support $\left(x_{1}, x_{2}, \cdots, x_{k}\right)$ to denote Support $(a)$ for an arc $a$ with coordinate $x=\left(x_{1}, x_{2}, \cdots, x_{k}\right)$. 
Example 2 Let $k=6$. If $\mathcal{P}(a)=\left\{P_{s_{1}, r_{1}}, P_{s_{2}, r_{3}}, P_{s_{2}, r_{5}}\right\}$, then the coordinate of $a$ is $(1,0,2,0,2,0)$ and $\operatorname{Support}(a)=\{1,3,5\}$.

Let $\mathcal{F}$ be a multi-flow with coefficient matrices $\left(c_{i, j}^{(\ell)}\right) \in \mathbb{R}^{k \times k}$ for $\ell=$ $1,2, \cdots, k$. Let $a$ be an arc with coordinate $x=\left(x_{1}, x_{2}, \cdots, x_{k}\right)$. Then, by definition,

$$
\begin{aligned}
f_{\ell}(a) & =\sum_{i, j=1}^{k} c_{i, j}^{(\ell)} f_{i, j}(a) \\
& =\sum_{j \in \operatorname{Support}(x)}^{k} c_{x_{j}, j}^{(\ell)} \\
& =\sum_{j=1}^{k} c_{x_{j}, j}^{(\ell)} .
\end{aligned}
$$

Note that in the above derivation and thereinafter, we assume $c_{0, j}^{(\ell)} \equiv 0$ for all $1 \leq \ell, j \leq k$.

Since $f_{\ell}(a)$ only depends on the coordinate of $a$, we also use $f_{\ell}(x)$ or $f_{\ell}\left(x_{1}, x_{2}, \cdots, x_{k}\right)$ to denote $f_{\ell}(a)$ for $\ell=1,2, \cdots k$ for an arc with coordinate $x=\left(x_{1}, x_{2}, \cdots, x_{k}\right)$. Similarly, we use $|\mathcal{F}|(x)$ or $|\mathcal{F}|\left(x_{1}, x_{2}, \cdots, x_{k}\right)$ to denote $|\mathcal{F}|(a)$. Denote by

$$
X=\left\{\left(x_{1}, x_{2}, \cdots, x_{k}\right) ; 0 \leq x_{i} \leq k, i=1,2, \cdots, k\right\}
$$

the set of all $(k+1)^{k}$ coordinates. We have:

Theorem 2 If $|\mathcal{F}|(x)<1$ for all $x \in X$, then $\mathcal{F}$ is feasible.

Example 3 The homogeneous multi-commodity flow $\mathcal{F}$ constructed in [4] is feasible. Note that the coefficient matrices of $\mathcal{F}$ satisfy

$$
c_{i, j}^{(\ell)}=\left\{\begin{array}{l}
\frac{2 k-3}{k(3 k-4)}, \text { if } i=j=\ell, \\
\frac{k-1}{k(3 k-4)}, \text { if } i=\ell ; j \neq \ell \text { or } j=\ell ; i \neq \ell, \\
\frac{-1}{k(3 k-4)}, i \neq \ell \text { and } j \neq \ell
\end{array}\right.
$$

for $\ell=1,2, \cdots, k$. Then for any $\left(x_{1}, x_{2}, \cdots, x_{k}\right) \in X$, if $x_{j}=j$,

$$
\begin{aligned}
\sum_{\ell=1}^{k}\left|c_{x_{j}, j}^{(\ell)}\right| & =\left|c_{j, j}^{(j)}\right|+\sum_{\ell \neq j}^{k}\left|c_{j, j}^{(\ell)}\right| \\
& =\frac{2 k-3}{k(3 k-4)}+\frac{k-1}{k(3 k-4)} \\
& =\frac{1}{k}
\end{aligned}
$$


and if $x_{j} \neq 0, j$,

$$
\begin{aligned}
\sum_{\ell=1}^{k}\left|c_{x_{j}, j}^{(\ell)}\right| & =\left|c_{x_{j}, j}^{(j)}\right|+\left|c_{x_{j}, j}^{\left(x_{j}\right)}\right|+\sum_{\ell \neq j, x_{j}}^{k}\left|c_{x_{j}, j}^{(\ell)}\right| \\
& =\frac{2(k-1)}{k(3 k-4)}+\frac{k-2}{k(3 k-4)} \\
& =\frac{1}{k} .
\end{aligned}
$$

Hence,

$$
\begin{aligned}
|\mathcal{F}|\left(x_{1}, x_{2}, \cdots, x_{k}\right) & =\sum_{\ell=1}^{k}\left|\sum_{j=1}^{k} c_{x_{j}, j}^{(\ell)}\right| \\
& \leq \sum_{j=1}^{k} \sum_{\ell=1}^{k}\left|c_{x_{j}, j}^{(\ell)}\right| \\
& =\sum_{j \in \operatorname{Support}(x)}\left(\sum_{\ell=1}^{k}\left|c_{x_{j}, j}^{(\ell)}\right|\right) \\
& \leq k \sum_{\ell=1}^{k}\left|c_{x_{j}, j}^{(\ell)}\right| \\
& =1 .
\end{aligned}
$$

By Theorem $2, \mathcal{F}$ is feasible.

\section{The Optimization Problem}

In this section, we are interested in the maximum $d$ for which there exists a feasible multi-flows constructed from the elementary flows $\mathbf{F}$ with rate $(d, d, \cdots, d)$, which naturally leads to the following optimization problem:

$$
\begin{array}{ll}
\underset{C_{1}, C_{2}, \cdots, C_{k}}{\operatorname{maximize}} & d \\
\text { subject to } & \sum_{\ell=1}^{k}\left|\sum_{j=1}^{k} c_{x_{j}, j}^{(\ell)}\right| \leq 1,0 \leq x_{j} \leq k,
\end{array}
$$

where $C_{1}=\left(c_{i, j}^{(1)}\right), C_{2}=\left(c_{i, j}^{(2)}\right), \cdots, C_{k}=\left(c_{i, j}^{(k)}\right)$ are $k \times k$ matrices such that 1) $\sum_{j=1}^{k} c_{i, j}^{(\ell)}=0$, for all $\left.1 \leq i \leq k, i \neq \ell ; 2\right) \sum_{i=1}^{k} c_{i, j}^{(\ell)}=0$, for all $1 \leq j \leq k$, $j \neq \ell ; 3) \sum_{i=1}^{k} \sum_{j=1}^{k} c_{i, j}^{(\ell)} \equiv d$ for $\ell=1,2, \cdots, k$. Recall that we assume $c_{0, j}^{(\ell)} \equiv 0$ for $1 \leq \ell, j \leq k$ in the above. It is equivalent to the following optimization 
problem:

$$
\begin{array}{ll}
\underset{C_{1}, C_{2}, \cdots, C_{k}}{\operatorname{minimize}} & c \\
\text { subject to } & \sum_{i=1}^{k} \sum_{j=1}^{k} c_{i, j}^{(\ell)} \equiv 1, \ell=1,2, \cdots, k,
\end{array}
$$

where $c=\max _{0 \leq x_{j} \leq k}\left\{\sum_{\ell=1}^{k}\left|\sum_{j=1}^{k} c_{x_{j}, j}^{(\ell)}\right|\right\}$ and $C_{1}=\left(c_{i, j}^{(1)}\right), C_{2}=\left(c_{i, j}^{(2)}\right), \cdots, C_{k}=$ $\left(c_{i, j}^{(k)}\right)$ are $k \times k$ matrices such that 1$) \sum_{j=1}^{k} c_{i, j}^{(\ell)}=0$, for all $1 \leq i \leq k, i \neq \ell$; 2) $\sum_{i=1}^{k} c_{i, j}^{(\ell)}=0$, for all $1 \leq j \leq k, j \neq \ell$.

The equivalence of the optimization problems (8) and (9) can be seen as follows. If $d_{0}$ is the solution of (8) achieved by $\left\{C_{\ell}=\left(c_{i, j}^{(\ell)}\right)\right\}_{\ell=1}^{k}$, then letting $\widetilde{C}_{\ell}=\left(\widetilde{c}_{i, j}^{(\ell)}\right)=\frac{1}{d_{0}} C_{\ell}$, we will have $\sum_{i=1}^{k} \sum_{j=1}^{k} \widetilde{c}_{i, j}^{(\ell)} \equiv 1$. Note that $\frac{1}{d_{0}}$ is the solution of the optimization problem (9) achieved by matrices $\widetilde{C}_{\ell}, \ell=$ $1,2, \cdots, k$. On the other hand, if $c_{0}$ is the solution of (9) achieved by $\left\{C_{\ell}=\right.$ $\left(c_{i, j}^{(\ell)}\right\}_{\ell=1}^{k}$, then letting $\widetilde{C}_{\ell}=\left(\widetilde{c}_{i, j}^{(\ell)}\right)=\frac{1}{c_{0}} C_{\ell}$, we will have $\sum_{\ell=1}^{k}\left|\sum_{j=1}^{k} c_{x_{j}, j}^{(\ell)}\right| \leq$ 1. Note that $\frac{1}{c_{0}}$ is the solution of the optimization problem (8) achieved by matrices $\widetilde{C}_{\ell}, \ell=1,2, \cdots, k$.

Let us consider the case of $k=2$. By the above-mentioned equivalence, we only need to consider the optimization problem (9). Note that for this case,

$$
C_{1}=\left(\begin{array}{rr}
p & 1-p \\
1-p & p-1
\end{array}\right), \quad C_{2}=\left(\begin{array}{cc}
q-1 & 1-q \\
1-q & q
\end{array}\right),
$$

where $p, q \in \mathbb{R}$. And there are in total $3^{2}=9$ coordinates:

$$
X=\{(0,0),(0,1),(0,2),(1,0),(1,1),(1,2),(2,0),(2,1),(2,2)\} .
$$

By definition, $|\mathcal{F}|\left(x_{1}, x_{2}\right)=\sum_{\ell=1}^{k}\left|\sum_{j=1}^{k} c_{x_{j}, j}^{(\ell)}\right|$ and we have $|\mathcal{F}|(0,0)=0$, $|\mathcal{F}|(0,1)=|\mathcal{F}|(2,0)=|1-p|+|1-q|,|\mathcal{F}|(0,2)=|1-p|+|q|,|\mathcal{F}|(1,0)=$ $|p|+|1-q|,|\mathcal{F}|(1,2)=2|p+q-1|,|\mathcal{F}|(2,1)=2(|1-p|+|1-q|),|\mathcal{F}|(1,1)=$ $|\mathcal{F}|(2,2)=1$.

Let

$$
\begin{aligned}
c(p, q) & =\max \{|\mathcal{F}|(x) ; x \in X\} \\
& =\max \{2(|1-p|+|1-q|),|p|+|1-q|,|1-p|+|q|, 2|p+q-1|, 1\}
\end{aligned}
$$

In the following, we will prove

$$
\min _{p, q \in \mathbb{R}} c(p, q)=1 .
$$

First, it is easy to verify that $c\left(\frac{3}{4}, \frac{3}{4}\right)=1$. Second, consider the following four cases:

1. If $p>\frac{3}{4}, q \geq \frac{3}{4}$, then $c(p, q) \geq 2|p+q-1|>1$;

2. If $p \leq \frac{3}{4}, q>\frac{3}{4}$, then $c(p, q) \geq|1-p|+|q|>1$; 
3. If $p<\frac{3}{4}, q \leq \frac{3}{4}$, then $c(p, q) \geq 2(|1-p|+|1-q|)>1$;

4. If $p \geq \frac{3}{4}, q<\frac{3}{4}$, then $c(p, q) \geq|p|+|1-q|>1$.

Hence, we have

Theorem 3 If $k=2$, then the optimization problem (8) has solution 1.

Clearly, if the optimization problems (8) has solution $d_{0} \geq 1$, then there exists a "constructed multi-flow" which can settle the M. Langberg and M. Médard's conjecture. However, when $k \geq 3$, this is not the case, as shown below.

Theorem 4 Let $k \geq 3$ and $d_{0}$ be the solution of the optimization problem (8). Then, $\frac{8}{9} \leq d_{0}<1$.

In the following two sections, we will prove Theorem 4.

\section{The Lower Bound}

In this section, we prove the lower bound part of Theorem 4.

We first introduce some notations. Let $x=\left(x_{1}, x_{2}, \cdots, x_{k}\right) \in X$ be a coordinate (recall that a coordinate satisfies $0 \leq x_{\ell} \leq k$, for $\ell=1,2, \cdots, k$ ). And define

$$
\alpha(x):=\left|\left\{x_{\ell} ; x_{\ell} \neq 0, \ell=1,2, \cdots, k\right\}\right| ;
$$

in other words, $\alpha(x)$ is the size of the support of $x$. Let $j_{1}, j_{2}, \cdots, j_{\alpha(a)}$ be the support of $x$. Then, we define

$$
\operatorname{Ind}_{x}:=\left\{x_{j_{1}}, j_{1}, x_{j_{2}}, j_{2}, \cdots, x_{j_{\alpha(a)}}, j_{\alpha(a)}\right\} .
$$

Note that $I n d_{x}$ is a multiset (an element in a multiset can occur more than once) and we call it the index of $x$. For any $\ell=1,2, \cdots, k$, denote by $m_{I n d_{x}}(\ell)$ the multiplicity of $\ell$ in $\operatorname{Ind}_{x}$ (if $\ell \notin I n d_{x}$, then $m_{I n d_{x}}(\ell)=0$ ). And we further define

$$
\beta(x):=\left|\left\{\ell ; m_{\text {Ind }_{a}}(\ell) \neq 0\right\}\right| \text {. }
$$

For example, if $x=(1,0,2,0,3)$, then $\alpha(x)=3, \operatorname{Ind}_{a}=\{1,1,2,3,3,5\}$, $m_{\text {Ind }}(1)=m_{\text {Ind }}(3)=2, m_{\text {Ind }}(2)=m_{\text {Ind }}(5)=1, m_{I n d_{x}}(4)=0$, and $\beta(x)=4$.

Now, we give the main result of this section.

Theorem 5 Let $k \geq 3$ and $d_{0}$ be the solution of the optimization problem (8). Then

$$
d_{0} \geq \frac{8}{9}
$$

Proof To this end, we consider the optimization problem (9) and prove its optimal solution $c_{0} \leq \frac{9}{8}$.

Let matrices $C_{\ell}^{*}=\left(c_{i, j}^{(\ell)}\right)$ be such that

$$
c_{i, j}^{(\ell)}=\left\{\begin{array}{l}
\frac{2}{k}-\frac{1}{k^{2}}, \text { if } i=j=\ell, \\
\frac{1}{k}-\frac{1}{k^{2}}, \text { if } i=\ell ; j \neq \ell \text { or } j=\ell ; i \neq \ell, \\
-\frac{1}{k^{2}}, \quad i \neq \ell \text { and } j \neq \ell,
\end{array}\right.
$$


for $\ell=1,2, \cdots, k$. It is easy to verify that $\sum_{i=1}^{k} \sum_{j=1}^{k} c_{i, j}^{(\ell)} \equiv 1$ for $\ell=$ $1,2, \cdots, k$. Recall that for $x=\left(x_{1}, x_{2}, \cdots, x_{k}\right) \in X$,

$$
f_{\ell}(x)=\sum_{j=1}^{k} c_{x_{j}, j}^{(\ell)}
$$

and

$$
|\mathcal{F}|(x)=\sum_{\ell=1}^{k}\left|f_{\ell}(x)\right|=\sum_{\ell=1}^{k}\left|\sum_{j=1}^{k} c_{x_{j}, j}^{(\ell)}\right| .
$$

We calculate $\max _{x \in X}\{|\mathcal{F}|(x)\}$. If $x=(0,0, \cdots, 0)$, then by definition, $|\mathcal{F}|(x)=$ 0 . So, in the following, we suppose $x \neq(0,0, \cdots, 0)$. Firstly, we calculate $f_{\ell}(x)$ for some fixed $\ell \in\{1,2, \cdots, k\}$. It not hard to check the following two cases:

1. $m_{\text {Ind }_{x}}(\ell)=0$. In this case, by the definition of $C_{\ell}^{*}$, we have

$$
f_{\ell}(x)=\frac{-\alpha(x)}{k^{2}}
$$

2. $m_{\operatorname{Ind}_{x}}(\ell) \neq 0$. In this case, by the definition of $C_{\ell}^{*}$, we have

$$
f_{\ell}(x)=\frac{k m_{I n d_{x}}(\ell)-\alpha(x)}{k^{2}} .
$$

Secondly, we calculate $|\mathcal{F}|(x)$. Noticing that $\sum_{\ell=1}^{k} m_{I n d_{x}}(\ell)=2 \alpha(x)$, we have

$$
\begin{aligned}
|\mathcal{F}|(x) & =\sum_{1 \leq \ell \leq k}\left|f_{\ell}(x)\right| \\
& =\sum_{m_{\text {Ind }}(\ell)=0}-f_{\ell}(x)+\sum_{m_{\text {Indx }}(\ell) \neq 0} f_{\ell}(x) \\
& =\frac{(k-\beta(x)) \alpha(x)}{k^{2}}+\frac{2 k \alpha(x)-\beta(x) \alpha(x)}{k^{2}} \\
& =\frac{3 k \alpha(x)-2 \beta(x) \alpha(x)}{k^{2}} .
\end{aligned}
$$

Note that $\alpha(x) \leq \beta(x)$, we have

$$
|\mathcal{F}|(x) \leq \frac{3 k \alpha(x)-2 \alpha^{2}(x)}{k^{2}}
$$

Consider the following quadratic function on $\alpha$

$$
h(\alpha):=\frac{\alpha(3 k-2 \alpha)}{k^{2}},
$$

and it is easy to see that it takes the maximum of $9 / 8$ when $\alpha=3 k / 4$. Hence, we have

$$
\max _{0 \leq x_{j} \leq k}\left\{\sum_{\ell=1}^{k}\left|\sum_{j=1}^{k} c_{x_{j}, j}^{(\ell)}\right|\right\} \leq \frac{9}{8},
$$

as desired. 
Remark 1 Since $\alpha(x)$ is an integer, the bound of Theorem 5 can be slightly improved to $d_{0}>\frac{8}{9}$ for $4 \nmid k$.

Example 4 Consider the case $k=3$, and let

$$
C_{1}^{*}=\left(\begin{array}{ccc}
\frac{5}{9} & \frac{2}{9} & \frac{2}{9} \\
\frac{2}{9} & -\frac{1}{9} & -\frac{1}{9} \\
\frac{2}{9} & -\frac{1}{9} & -\frac{1}{9}
\end{array}\right) ; C_{2}^{*}=\left(\begin{array}{ccc}
-\frac{1}{9} & \frac{2}{9} & -\frac{1}{9} \\
\frac{2}{9} & \frac{5}{9} & \frac{2}{9} \\
-\frac{1}{9} & \frac{2}{9} & -\frac{1}{9}
\end{array}\right) ; C_{3}^{*}=\left(\begin{array}{ccc}
-\frac{1}{9} & -\frac{1}{9} & \frac{2}{9} \\
-\frac{1}{9} & -\frac{1}{9} & \frac{2}{9} \\
\frac{2}{9} & \frac{2}{9} & \frac{5}{9}
\end{array}\right)
$$

By Equation (13), we can see that $|\mathcal{F}|(x)$ takes its maximum value when $\alpha(x)=\beta(x)=2$. For example, when $x=(1,2,0)$, we have $\alpha(x)=\beta(x)=2$ and further

$$
\begin{aligned}
|\mathcal{F}|(1,2,0) & =\left|f_{1}(1,2,0)\right|+\left|f_{2}(1,2,0)\right|+\left|f_{3}(1,2,0)\right| \\
& =\left|\frac{5}{9}+\frac{2}{9}\right|+\left|-\frac{1}{9}+\frac{2}{9}\right|+\left|-\frac{1}{9}-\frac{1}{9}\right| \\
& =\frac{10}{9}<\frac{9}{8}
\end{aligned}
$$

Hence, for $k=3, d_{0} \geq \frac{9}{10}>\frac{8}{9}$.

The proof of Theorem 5 implies the following corollary.

Corollary 1 Let $\mathcal{N}$ be a strongly reachable network. If the coordinate $x$ of any arc a satisfies one of the following conditions:

1. $\alpha(x) \leq \frac{8}{9} \beta(x)$;

2. $\alpha(x)=k$;

3. $\alpha(x) \leq k / 2$,

then $\overline{\mathcal{N}}$ has a feasible multi-flow with rate $(1,1, \cdots, 1)$.

Proof Given Condition 1), one can have $|\mathcal{F}|(x) \leq 1$ from (13) via straightforward computations. Simple inspections on Equation (15) will reveal that Condition 2) or 3) will imply $h(\alpha) \leq 1$ and thus $|\mathcal{F}|(x) \leq 1$.

To complete this section, we conjecture that the solution of the optimization problem (9) is achieved by $C_{1}^{*}, C_{2}^{*}, \cdots, C_{k}^{*}$ constructed in the proof of Theorem 5.

\section{The Upper Bound}

In this section, we prove the upper bound part of Theorem 4 .

To this end, we consider the optimization problem (9). Let $C_{1}=\left(c_{i, j}^{(1)}\right), C_{2}=$ $\left(c_{i, j}^{(2)}\right), \cdots, C_{k}=\left(c_{i, j}^{(k)}\right)$ be $k \times k$ matrices such that $(1) \sum_{j=1}^{k} c_{i, j}^{(\ell)}=0$, for all $1 \leq i \leq k, i \neq \ell ;(2) \sum_{i=1}^{k} c_{i, j}^{(\ell)}=0$, for all $1 \leq j \leq k, j \neq \ell$ and (3) 
$\sum_{i=1}^{k} \sum_{j=1}^{k} c_{i, j}^{(\ell)} \equiv 1, \quad \ell=1,2, \cdots, k$. Let $X=\left\{\left(x_{1}, x_{2}, \cdots, x_{k}\right) ; 0 \leq x_{i} \leq\right.$ $k, i=1,2, \cdots, k\}$ be the set of coordinates. Recall that

$$
c=\max _{x \in X}\left\{|\mathcal{F}|(x)=\sum_{\ell=1}^{k}\left|f_{i}(x)\right|\right\}=\max _{0 \leq x_{j} \leq k}\left\{\sum_{\ell=1}^{k}\left|\sum_{j=1}^{k} c_{x_{j}, j}^{(\ell)}\right|\right\}
$$

In the following, we use $c_{0}$ to denote the solution of (9) and prove $c_{0}>1$. Note that if $c_{0} \leq 1$, then the solution of $(8) d_{0} \geq 1$.

We first give a simple but useful result.

Lemma 2 If $c_{0} \leq 1$, then for all $\ell, i, j=1,2, \cdots, k,\left|c_{i, j}^{(\ell)}\right| \leq 1$.

Proof Suppose, by way of contradiction, that there exist $1 \leq \ell, i, j \leq k$, such that $\left|c_{i, j}^{(\ell)}\right|>1$. Then, consider the coordinate $x=\left(x_{1}, x_{2}, \cdots, x_{j}, \cdots, x_{k}\right)$ such that

$$
x_{r}=\left\{\begin{array}{l}
0, r \neq j \\
i, r=j
\end{array}\right.
$$

Note that

$$
|\mathcal{F}|(x)=\sum_{r=1}^{k}\left|f_{r}(x)\right| \geq\left|f_{\ell}(x)\right|=\left|c_{i, j}^{(\ell)}\right|>1,
$$

which is however contradictory to the fact that $c_{0} \leq 1$.

Lemma 3 If $c_{0} \leq 1$, then for all $\ell, j=1,2, \cdots, k$, we have $c_{\ell, j}^{(\ell)} \geq c_{i, j}^{(\ell)}$, if $i \neq \ell$.

Proof Suppose, on the contrary, that there exist $1 \leq \ell \leq k$ and $1 \leq j \leq k$, such that $c_{i, j}^{(\ell)}>c_{\ell, j}^{(\ell)}$ for some $i \neq \ell$. Then, consider the coordinate $x=$ $\left(x_{1}, x_{2}, \cdots, x_{j}, \cdots, x_{k}\right)$ such that

$$
x_{r}=\left\{\begin{array}{l}
\ell, r \neq j \\
i, r=j
\end{array}\right.
$$

As before, we have

$$
\begin{aligned}
|\mathcal{F}|(x) & =\sum_{r=1}^{k}\left|f_{r}(x)\right| \\
& \geq\left|f_{\ell}(x)\right| \\
& =\left|c_{\ell, 1}^{(\ell)}+c_{\ell, 2}^{(\ell)}+\cdots+c_{\ell, j-1}^{(\ell)}+c_{i, j}^{(\ell)}+c_{\ell, j+1}^{(\ell)}+\cdots c_{\ell, k}^{(\ell)}\right| \\
& \geq c_{\ell, 1}^{(\ell)}+c_{\ell, 2}^{(\ell)}+\cdots+c_{\ell, j-1}^{(\ell)}+c_{i, j}^{(\ell)}+c_{\ell, j+1}^{(\ell)}+\cdots c_{\ell, k}^{(\ell)} \\
& >c_{\ell, 1}^{(\ell)}+c_{\ell, 2}^{(\ell)}+\cdots+c_{\ell, j-1}^{(\ell)}+c_{\ell, j}^{(\ell)}+c_{\ell, j+1}^{(\ell)}+\cdots c_{\ell, k}^{(\ell)} \\
& =1,
\end{aligned}
$$

which is however contradictory to the fact that $c_{0} \leq 1$. 
Corollary 2 If $c_{0} \leq 1$, then for all $\ell=1,2, \cdots, k$ and $j=1,2, \cdots, k, c_{\ell, j}^{(\ell)} \geq$ 0 .

Proof Since otherwise $\sum_{i=1}^{k} c_{i, j}^{(\ell)} \leq k c_{\ell, j}^{(\ell)}<0$, which contradicts Theorem 1

Corollary 3 If $c_{0} \leq 1$, then for all $\ell=1,2, \cdots, k, c_{\ell, \ell}^{(\ell)} \geq \frac{1}{k}$.

Proof Since otherwise $\sum_{j=1}^{k} c_{i, j}^{(\ell)} \leq k c_{\ell, j}^{(\ell)}<1$, which contradicts Theorem 1

The following theorem says that if $c_{0} \leq 1$, then $c_{i, j}^{(i)}$ and $c_{i, j}^{(j)}$ have the same absolute value and furthermore, except the $\ell$-th row, the $\ell$-th column and the diagonal, all the other positions of $C^{(\ell)}$ must be zero.

Theorem 6 If $c_{0} \leq 1$, then for all $i \neq j, c_{i, j}^{(i)}=\left|c_{i, j}^{(j)}\right|$ and $c_{i, j}^{(\ell)}=0$, for all $\ell \neq i, j$.

Proof (1) We first prove for all $\ell=1,2, \cdots, k$, and $j=1,2, \cdots, k, c_{\ell, j}^{(\ell)} \geq$ $\sum_{i \neq \ell}\left|c_{\ell, j}^{(i)}\right|$.

Taking the coordinate $(0,1,1, \cdots, 1)$, we have, by Theorem 1 , that

$$
\begin{aligned}
|\mathcal{F}|(0,1,1, \cdots, 1) & =\sum_{r=1}^{k}\left|f_{r}(0,1,1, \cdots, 1)\right| \\
& =\sum_{r=1}^{k}\left|c_{1,2}^{(r)}+c_{1,3}^{(r)}+\cdots+c_{1, k}^{(r)}\right| \\
& =1-c_{1,1}^{(1)}+\left|c_{1,1}^{(2)}\right|+\cdots+\left|c_{1,1}^{(k)}\right| .
\end{aligned}
$$

Since $c_{0} \leq 1$, we have $|\mathcal{F}|(0,1,1, \cdots, 1) \leq 1$. Hence, the above equation implies

$$
c_{1,1}^{(1)} \geq\left|c_{1,1}^{(2)}\right|+\left|c_{1,1}^{(3)}\right|+\cdots+\left|c_{1,1}^{(k)}\right| .
$$

By taking the coordinate $(1,0,1, \cdots, 1)$, we have

$$
|\mathcal{F}|(1,0,1, \cdots, 1)=\sum_{r=1}^{k}\left|f_{r}(1,0,1, \cdots, 1)\right|=1-c_{1,2}^{(1)}+\left|c_{1,2}^{(2)}\right|+\cdots+\left|c_{1,2}^{(k)}\right| .
$$

Hence,

$$
c_{1,2}^{(1)} \geq\left|c_{1,2}^{(2)}\right|+\left|c_{1,2}^{(3)}\right|+\cdots+\left|c_{1,2}^{(k)}\right|
$$

More generally, by taking coordinates $(1,1,0,1 \cdots, 1),(1,1,1,0,1 \cdots, 1), \cdots$, and $(1,1, \cdots, 1,0)$, we have: for all $j=1,2, \cdots, k$,

$$
c_{1, j}^{(1)} \geq\left|c_{1, j}^{(2)}\right|+\left|c_{1, j}^{(3)}\right|+\cdots+\left|c_{1, j}^{(k)}\right| .
$$

Now consider the coordinates $(0,2,2, \cdots, 2),(2,0,2, \cdots, 2), \cdots$ and $(2,2, \cdots, 2,0)$. Similarly, we can have: for all $j=1,2, \cdots, k$,

$$
c_{2, j}^{(2)} \geq\left|c_{2, j}^{(1)}\right|+\left|c_{2, j}^{(3)}\right|+\cdots+\left|c_{2, j}^{(k)}\right| .
$$


And more generally, by taking the coordinates $(0, \ell, \ell, \cdots, \ell),(\ell, 0, \ell, \cdots, \ell), \cdots$ and $(\ell, \ell, \cdots, \ell, 0)$, we have: for all $\ell=1,2, \cdots, k$, and $j=1,2, \cdots, k$,

$$
c_{\ell, j}^{(\ell)} \geq\left|c_{\ell, j}^{(1)}\right|+\cdots+\left|c_{\ell, j}^{(\ell-1)}\right|+\left|c_{\ell, j}^{(\ell+1)}\right|+\cdots+\left|c_{\ell, j}^{(k)}\right|=\sum_{i \neq \ell}\left|c_{\ell, j}^{(i)}\right| .
$$

(2) We prove that for all $i \neq j c_{i, j}^{(i)} \geq\left|c_{i, j}^{(j)}\right|$, and "=" holds if and only for all $\ell \in\{1,2, \cdots, k\} \backslash\{i, j\}, c_{i, j}^{(\ell)}=0$.

By Equation (19), we have $c_{1,2}^{(1)} \geq\left|c_{1,2}^{(2)}\right|$, and "=" holds if and only if

$$
\left|c_{1,2}^{(3)}\right|=\left|c_{1,2}^{(4)}\right|=\cdots=\left|c_{1,2}^{(k)}\right|=0,
$$

which means that for all $\ell \in\{1,2, \cdots, k\} \backslash\{1,2\}, c_{1,2}^{(\ell)}=0$.

Similarly, by Equation (20), we have

$$
c_{1,2}^{(1)} \geq\left|c_{1,2}^{(2)}\right|, \quad c_{1,3}^{(1)} \geq\left|c_{1,3}^{(3)}\right|, \quad \ldots, \quad c_{1, k}^{(1)} \geq\left|c_{1, k}^{(k)}\right|,
$$

where in the $(j-1)$-th inequality, the "=" holds if and only if for all $\ell \in$ $\{1,2, \cdots, k\} \backslash\{1, j\}, c_{1, j}^{(\ell)}=0$.

In the same way, by Equation (21), we have: for $j=1,3, \cdots, k, c_{2, j}^{(2)} \geq\left|c_{2, j}^{(j)}\right|$ and the "=" holds if and only if for all $\ell \in\{1,2, \cdots, k\} \backslash\{2, j\}, c_{2, j}^{(\ell)}=0$. And, by Equation (22) we have: for all $i \neq j$,

$$
c_{i, j}^{(i)} \geq\left|c_{i, j}^{(j)}\right|,
$$

where "=" holds if and only for all $\ell \in\{1,2, \cdots, k\} \backslash\{i, j\}, c_{i, j}^{(\ell)}=0$.

(3) We prove that for all $i \neq j, c_{i, j}^{(i)}=\left|c_{i, j}^{(j)}\right|$ and hence for all $\ell \in\{1,2, \cdots, k\} \backslash$ $\{i, j\}, c_{i, j}^{(\ell)}=0$.

Adding all inequalities of (23) together and then adding $\sum_{\ell=1}^{k} c_{\ell, \ell}^{(\ell)}$ to both sides, we have

$$
\sum_{i=1}^{k} c_{i, i}^{(i)}+\sum_{i \neq j} c_{i, j}^{(i)} \geq \sum_{j=1}^{k} c_{j, j}^{(j)}+\sum_{i \neq j}\left|c_{i, j}^{(j)}\right| .
$$

By Theorem 1, we have

$$
\sum_{i=1}^{k} c_{i, i}^{(i)}+\sum_{i \neq j} c_{i, j}^{(i)}=\sum_{i=1}^{k}\left(\sum_{j=1}^{k} c_{i, j}^{(i)}\right)=k
$$

and

$$
\sum_{j=1}^{k} c_{j, j}^{(j)}+\sum_{i \neq j}\left|c_{i, j}^{(j)}\right| \geq \sum_{j=1}^{k} c_{j, j}^{(j)}+\sum_{i \neq j} c_{i, j}^{(j)}=\sum_{j=1}^{k}\left(\sum_{i=1}^{k} c_{i, j}^{(j)}\right)=k .
$$

Hence,

$$
\sum_{i \neq j} c_{i, j}^{(i)} \leq \sum_{i \neq j}\left|c_{i, j}^{(j)}\right| \cdot
$$

By the result of step (2), the equality of (25) holds and hence for all $\ell \in$ $\{1,2, \cdots, k\} \backslash\{i, j\}, c_{i, j}^{(\ell)}=0$. The proof is then complete. 
Corollary 4 For $i=1,2, \cdots, k$ and for all $\ell \neq i$,

$$
c_{i, \ell}^{(\ell)}=c_{\ell, i}^{(\ell)}=-c_{i, i}^{(\ell)} \text {. }
$$

Proof By Theorem 1, we have

$$
c_{i, 1}^{(\ell)}+c_{i, 2}^{(\ell)}+\cdots+c_{i, k}^{(\ell)}=0 .
$$

And, by Theorem $6, c_{i, j}^{(\ell)} \neq 0$ if and only if $j=i$ or $j=\ell$. Thus we have $c_{i, i}^{(\ell)}+c_{i, \ell}^{(\ell)}=0$. Similarly, since

$$
c_{1, i}^{(\ell)}+c_{2, i}^{(\ell)}+\cdots+c_{k, i}^{(\ell)}=0,
$$

by Theorem 6 , we have $c_{i, i}^{(\ell)}+c_{\ell, i}^{(\ell)}=0$.

The above two cases complete the proof.

Remark 2 Combining with Corollary 2, we have that for $i=1,2, \cdots, k$ and for all $\ell \neq i, c_{i, \ell}^{(\ell)} \geq 0, c_{\ell, i}^{(\ell)} \geq 0$ and $c_{i, i}^{(\ell)} \leq 0$.

The following result further strengthens Corollary 3.

Lemma 4 If $c_{0} \leq 1$, then for all $\ell=1,2, \cdots, k$,

$$
c_{\ell, \ell}^{(\ell)} \geq \frac{1}{2}
$$

Proof By Equation (22) in the proof of Theorem 6, we have

$$
c_{\ell, \ell}^{(\ell)} \geq \sum_{j \neq \ell}\left|c_{\ell, \ell}^{(j)}\right| .
$$

By Corollary 4, for $j \neq \ell,\left|c_{\ell, \ell}^{(j)}\right|=\left|c_{\ell, j}^{(j)}\right|$. Plugging in the above inequality and using Theorem 6 , we have

$$
c_{\ell, \ell}^{(\ell)} \geq \sum_{j \neq \ell}\left|c_{\ell, j}^{(j)}\right|=\sum_{j \neq \ell} c_{\ell, j}^{(\ell)} .
$$

On the other hand, By Theorem 1, we have

$$
c_{\ell, \ell}^{(\ell)}+\sum_{j \neq \ell} c_{\ell, j}^{(\ell)}=\sum_{j=1}^{k} c_{\ell, j}^{(\ell)}=1
$$

Combining Equations (27) and (28) together, we have $c_{\ell, \ell}^{(\ell)} \geq \frac{1}{2}$, which completes the proof.

We are now ready to prove the following theorem.

Theorem 7 If $c_{0} \leq 1$, then for all $\ell=1,2, \cdots, k$,

$$
c_{\ell, \ell}^{(\ell)}=\frac{1}{2} \text {. }
$$


Proof Let $x^{1}, x^{2}, \cdots, x^{k}$ be coordinates such that $x^{i}=\left(x_{1}^{i}, x_{2}^{i}, \cdots, x_{k}^{i}\right)$, where

$$
x_{j}^{i}=\left\{\begin{array}{l}
j, j \neq i \\
0, i=j
\end{array}\right.
$$

That is to say, $x^{1}=(0,2,3, \cdots, k), x^{2}=(1,0,3, \cdots, k), \cdots, x^{k}=(1,2, \cdots, k-$ $1,0)$.

By definition, for all $\ell, i=1,2, \cdots, k$,

$$
f_{\ell}\left(x^{i}\right)=\sum_{j \neq i} c_{j, j}^{(\ell)}
$$

hence we have

$$
\sum_{i=1}^{k}|\mathcal{F}|\left(x^{i}\right)=\sum_{i=1}^{k} \sum_{\ell=1}^{k}\left|f_{\ell}\left(x^{i}\right)\right|=\sum_{\ell=1}^{k}\left(\sum_{i=1}^{k}\left|f_{\ell}\left(x^{i}\right)\right|\right)=\sum_{\ell=1}^{k}\left(\sum_{i=1}^{k}\left|\sum_{j \neq i} c_{j, j}^{(\ell)}\right|\right) .
$$

By Inequality (26), we can drop the absolute value operation and have that for all $\ell=1,2, \cdots, k$,

$$
\begin{aligned}
\sum_{i=1}^{k}\left|\sum_{j \neq i} c_{j, j}^{(\ell)}\right| & =\sum_{i \neq \ell} \sum_{j \neq i} c_{j, j}^{(\ell)}-\sum_{j \neq \ell} c_{j, j}^{(\ell)} \\
& =(k-1) c_{\ell, \ell}^{(\ell)}+(k-2) \sum_{j \neq \ell} c_{j, j}^{(\ell)}-\sum_{j \neq \ell} c_{j, j}^{(\ell)} \\
& =(k-1) c_{\ell, \ell}^{(\ell)}+(k-3) \sum_{j \neq \ell} c_{j, j}^{(\ell)} \\
& =(k-1) c_{\ell, \ell}^{(\ell)}-(k-3)\left(1-c_{\ell, \ell}^{(\ell)}\right) \\
& =(2 k-4) c_{\ell, \ell}^{(\ell)}-(k-3),
\end{aligned}
$$

where the 4 -th equality is obtained by Remark 2 .

Notice that $c_{0} \leq 1$, we have

$$
\sum_{i=1}^{k}|\mathcal{F}|\left(x^{i}\right)=\sum_{\ell=1}^{k}\left[(2 k-4) c_{\ell, \ell}^{(\ell)}-(k-3)\right]=(2 k-4) \sum_{\ell=1}^{k} c_{\ell, \ell}^{(\ell)}-k(k-3) \leq k,
$$

which implies

$$
\sum_{\ell=1}^{k} c_{\ell, \ell}^{(\ell)} \leq \frac{k}{2}
$$

By Lemma 4, we have that for $\ell=1,2, \cdots, k, c_{\ell, \ell}^{(\ell)}=\frac{1}{2}$, which completes the proof.

Corollary 5 If $c_{0} \leq 1$, then for $\ell=1,2, \cdots, k$,

$$
\sum_{j=1}^{k} c_{j, j}^{(\ell)}=0
$$


Proof By Corollary 4, we have

$$
\sum_{j=1}^{k} c_{j, j}^{(\ell)}=c_{\ell, \ell}^{(\ell)}-\sum_{j \neq \ell} c_{\ell, j}^{(\ell)}=c_{\ell, \ell}^{(\ell)}-\left(1-c_{\ell, \ell}^{(\ell)}\right)=0 .
$$

The corollary then immediately follows from Theorem 7.

Now, we can prove the main result in this section.

Theorem 8 Let $k \geq 3$ and $d_{0}$ be the solution of the optimization problem (8). Then

$$
d_{0}<1 \text {. }
$$

Proof It is sufficient to prove $c_{0}>1$. To this end, we suppose $c_{0} \leq 1$, which will lead to a contradiction.

Let $\bar{X}=\left(x_{i, j}\right)_{(k-1) \times k}$ be a matrix such that for $j=1,2, \cdots, k$, the $j$-th column of $\bar{X}$ is a permutation of integers $\{1,2, \cdots, k\} \backslash\{j\}$. By this definition, we see that $x_{i, j} \neq j$ for all $i=1,2, \cdots, k-1$. Hence, by Remark $2, c_{x_{i, j}, j}^{(\ell)} \geq 0$ for all $\ell=1,2, \cdots, k$.

Let $\overline{x_{i}}=\left(x_{i, 1}, x_{i, 2}, \cdots, x_{i, k}\right)$ be the $i$-th row of $X$. By the above discussion, for each $\ell=1,2, \cdots, k$, we have

$$
\begin{aligned}
\left|f_{\ell}\left(\overline{x_{i}}\right)\right| & =\left|f_{\ell}\left(x_{i, 1}, x_{i, 2}, \cdots, x_{i, k}\right)\right| \\
& =\left|c_{x_{i, 1}, 1}^{(\ell)}+c_{x_{i, 2}, 2}^{(\ell)}+\cdots+c_{x_{i, k}, k}^{(\ell)}\right| \\
& =c_{x_{i, 1}, 1}^{(\ell)}+c_{x_{i, 2}, 2}^{(\ell)}+\cdots+c_{x_{i, k}, k}^{(\ell)} \\
& =f_{\ell}\left(\overline{x_{i}}\right) .
\end{aligned}
$$

Note that for all $j=1,2, \cdots, k$, the $j$-th column of $\bar{X}$ is a permutation of $\{1,2, \cdots, k\} \backslash\{j\}$. Hence,

$$
\sum_{i=1}^{k-1}\left|f_{\ell}\left(\overline{x_{i}}\right)\right|=\sum_{i=1}^{k-1} f_{\ell}\left(\overline{x_{i}}\right)=\sum_{r, s: r \neq s} c_{r, s}^{(\ell)} .
$$

By Corollary 5, we have

$$
\begin{aligned}
\sum_{i=1}^{k-1}|\mathcal{F}|\left(\overline{x_{i}}\right) & =\sum_{i=1}^{k-1} \sum_{\ell=1}^{k}\left|f_{\ell}\left(\overline{x_{i}}\right)\right| \\
& =\sum_{\ell=1}^{k}\left(\sum_{i=1}^{k-1}\left|f_{\ell}\left(\overline{x_{i}}\right)\right|\right) \\
& =\sum_{\ell=1}^{k}\left(\left[\sum_{r, s: r \neq s} c_{r, s}^{(\ell)}+\sum_{s=1}^{k} c_{s, s}^{(\ell)}\right]-\sum_{s=1}^{k} c_{s, s}^{(\ell)}\right) \\
& =\sum_{\ell=1}^{k}\left(\sum_{r, s=1}^{k} c_{r, s}^{(\ell)}\right) .
\end{aligned}
$$

Note that if $c_{0} \leq 1$, then $\sum_{i=1}^{k-1}|\mathcal{F}|\left(\overline{x_{i}}\right) \leq k-1$, which is contradictory to the assumption that $\sum_{\ell=1}^{k}\left(\sum_{r, s=1}^{k} c_{r, s}^{(\ell)}\right)=k$. The proof is then complete. 


\section{References}

1. Z. Li and B. Li (2004) Network coding: The case of multiple unicast sessions. Proc. 42nd Annual Allerton:11-20

2. Y. Yang, X. Yin, X. Chen, Y. Yang and Z. Li (2014) A note on the multiple-unicast network coding conjecture. IEEE Communications Letters 18(5):869-872

3. R. Ahlswede, N. Cai, S. -Y. R. Li and R. Yeung (2000) Network information flow. IEEE Trans. Inf. Theory 46(4):1204-1216

4. M. Langberg and M. Médard (2009) On the multiple unicast network coding conjecture. Proc. 47th Annual Allerton:222-227

5. A. Schrijver (2003) Combinatorial Optimization. Springer-Verlag 